\title{
SCIPION, a new flexible ionospheric sounder in Senegal
}

\author{
Y. M. Le Roux, J. Ménard, J. P. Jolivet, P. J. Davy \\ France Telecom - CNET, F-22307 Lannion, France \\ Received: 16 December 1997 / Revised: 5 January 1998 / Accepted: 21 January 1998
}

\begin{abstract}
SCIPION is a new state of the art digital sounder that has been developed by France TelecomCNET for ionospheric monitoring and research. Extensive data processing using DSP technology has resulted in a low power, low cost and full featured system for both vertical and oblique soundings. A SCIPION system is in the process of being installed in Dakar, Senegal, to study HF propagation in the sub-equatorial ionosphere. However, preliminary results have still been obtained during experiments wit a prototype system. In this paper, the system is described and some illustrative examples of its capabilities are shown.
\end{abstract}

Key words. Ionosphere (Equatorial ionosphere, Instruments and Techniques) · Radio science (ionospheric propagation).

\section{Introduction}

Vertical and oblique HF sounders have proved to be particularly relevant for ionospheric studies. In the past, until the 1970s, most of sounding techniques were based on the transmission of high power pulses as it was the case for the US C-4 sounder, the Australian IPS-sounders and the Cossor system in Canada. Coded signals and frequency modulated waveforms were later introduced in order to save power while keeping acceptable range resolution (Wright and Pitteway, 1982; Bibl and Reinisch, 1978; Barry, 1971).

The ionospheric oblique/vertical sounder described in the paper use M-PSK coded waveforms, associated with specific digital signal processing techniques, which permits accurate measurements of the HF channel to be performed while using low transmitted power levels. It is the most recent version of systems that have been operated since the beginning of the 1980s, over various links (Le Roux et al., 1984; Le Roux et al. 1987),

Depending on options, the SCIPION sounder can perform the following measurements:
- Vertical sounding.

- Oblique sounding with time synchronization.

- Scattering function measurements.

- Polarization filtering.

- Angle of arrival measurements.

- Coherence analysis between spaced antenna while using only one receiver.

- Noise level or spectrum measurements.

- Impulse response sequences acquisition.

Furthermore, the impulse response sequences of the ionospheric channel measured by the system can be stored and later used in a channel reproducer for simulation purposes (Le Roux et al., 1990).

\section{The SCIPION ionospheric sounder}

Figure 1 summarizes the salient features and main performances of SCIPION.

\subsection{System components}

$T x / R x$ units. The transmitter and the receiver are integrated either into one single $\mathrm{Tx} / \mathrm{Rx}$ unit (monostatic option) or into two separate units (bistatic option). In the Tx unit, a synthesizer using direct digital synthesis (DDS) provides frequency generation. The HF transmitted signals are subsequently generated by modulation and frequency conversion. The RX unit comprises two HF heads with computercontrolled gains and phases. In normal operations, these two heads are associated with cross-polarized antennas to allow for $\mathrm{O}$ and $\mathrm{X}$ mode separation.

By means of a switching device, up to 8 receiving antennas can be used as an option. In this case, the antennas are sequentially scanned so that only one receiver is needed. This reduces cost and calibration problems. The minimum duration of analysis per antenna is $10 \mathrm{~ms}$.

A $25 \mathrm{~W}$ Max amplifier is found in a separate unit so as to be located in the vicinity of the antenna if needed. 


\begin{tabular}{|ll|}
\hline HF SIGNAL & $10 \%$ to $100 \%$ \\
Duty cycle & $2-4$ PSK \\
Modulation & programmable $( \pm 6.25 \mathrm{kHz}$ \\
Instantaneous bandwidth & from 1 to $30 \mathrm{MHz}$ \\
Frequency range & sequential, interleaved, random \\
Frequency hopping & from $30 \mathrm{sec}$ to $3 \mathrm{~min}$ \\
Duration of frequency scan & $0.005 \mathrm{PPM}$ \\
Frequency stability & \\
TRANSMITTER & manually or remotely (modem) \\
Fully programmable & $25 \mathrm{~W}$ average \\
Output power maximum & $50 \Omega$ \\
Output impedance & \\
RECEIVER & up to 16 antennas \\
Switching device & $50 \Omega$ \\
Input impedance & phase and gain programmable \\
2 HF input channels & $<15 \mathrm{~dB}$ \\
Noise factor & $>100 \mathrm{~dB}$ \\
Input range & $>90 \mathrm{~dB}$ \\
Image frequency rejection & $<20 \mathrm{kHz}$ \\
IF output & \\
\hline
\end{tabular}

Fig. 1. Performance specifications

Control unit. A Pentium PC provides control of the sounder operations, display of the results and data storage. Plug-in cards are added to the PC to permit control and synchronization of the sounder units and to provide the necessary signal processing capabilities.

As an option to the bistatic version, modems can be used for remote maintenance and/or control through telephone lines. In this case, the whole operating modes are controlled from one site (e.g. the Rx site).

The synchronization of the Tx and Rx units is provided by a plug-in card associated with a Global Positioning System (GPS) receiver. This permits the system to be operated everywhere in the world.

A signal-processing card provides digitization and processing of the analog signals from the Rx unit.

An MS-WINDOWS based graphical interface with mouse and on-line help in provided to visualize the data. Capabilities exist to visualize measurements either in realtime or in the form of animations for stored data.

Virtually any mass storage unit compatible with the PC can be used to store the data. Presently, an external 1 GByte disk unit permits the recording of several months of data in routine operations (4 ionograms per hour).

Antennas. Aerials must be broad bandwidth (1.5 to 30 $\mathrm{MHz}$ ) and have a radiation pattern appropriate to the current option (oblique or vertical). For vertical or quasi-vertical systems, DELTA type antennas are appropriate for both transmission and reception. Two cross-polarized antennas can be used at reception to perform O/X mode separation. For oblique systems, inverted-V are appropriate at both the transmitter and receiver sites. Loop antennas can also be particularly convenient as receiver antennas.

\begin{tabular}{|ll|}
\hline \multicolumn{2}{|l|}{ DIGITAL SIGNAL PROCESSING } \\
A/D conversion & $16 \mathrm{bits}$ \\
DSP & $60 \mathrm{MFlops}$ \\
Signal processing gain & 20 to $42 \mathrm{~dB}$ \\
Group delay range & 0 to $50 \mathrm{~ms}$ \\
Group delay spread/resolution & up to $8 \mathrm{~ms} / 0.5 \mathrm{~ms}$ \\
Max number of range bins & 256 \\
Range resolution & $>3 \mathrm{~km}$ \\
Doppler range/resolution & $\pm 1 \mathrm{~Hz}$ to $\pm 30 \mathrm{~Hz} / 10 \mathrm{mHz}$ typical \\
Noise & Spectrum $v$ s. sounding frequency \\
Polarization filtering & gain and phase adjustable \\
& \\
ADD ON SOFTWARES AND FACILITIES \\
Windows application & \\
On line help & \\
Vertical to oblique conversion & \\
Regional predictions & \\
Real- heights & \\
Angles of arrival : skymap & \\
Spatial coherency
\end{tabular}

\subsection{Operating modes and data display}

The operating modes are the following:

- Sweep or fixed frequency.

- Frequency scanning according to a selected program.

- Vertical incidence soundings (monostatic option).

- Quasi-vertical or oblique soundings (bistatic option)

All operating modes are computer controlled and can be activated according to any user-defined schedule. Depending on the SCIPION version and by means of the MS-WINDOWS graphic interface, the following data displays are provided:

- Amplitude ionogram (virtual heights vs. sounding frequency with color-coded echo amplitudes).

- Doppler ionogram (virtual heights vs. sounding frequency with color-coded Doppler shifts).

- Scattering function (virtual heights vs. Doppler shift with color-coded echo amplitudes).

- Noise spectrum vs. sounding frequency.

- Angles of arrival and their time evolutions (skymap).

- Coherence functions of received signals using several antennas.

\section{Examples of experimental results}

\subsection{Vertical ionograms and oblique extrapolations}

Figures 2 shows an ionogram measured with a prototype of the SCIPION sounder, in Dakar. For this ionogram, the frequency sweep duration was $100 \mathrm{~s}$, whereas the frequency step was $100 \mathrm{kHz}$. The relative amplitudes of the echoes are indicated by colors. It can be noticed that significant spread-F appears on the ionogram. This is a typical feature of the equatorial ionosphere which is known to severely affect $\mathrm{HF}$ transmissions in these areas. On the 


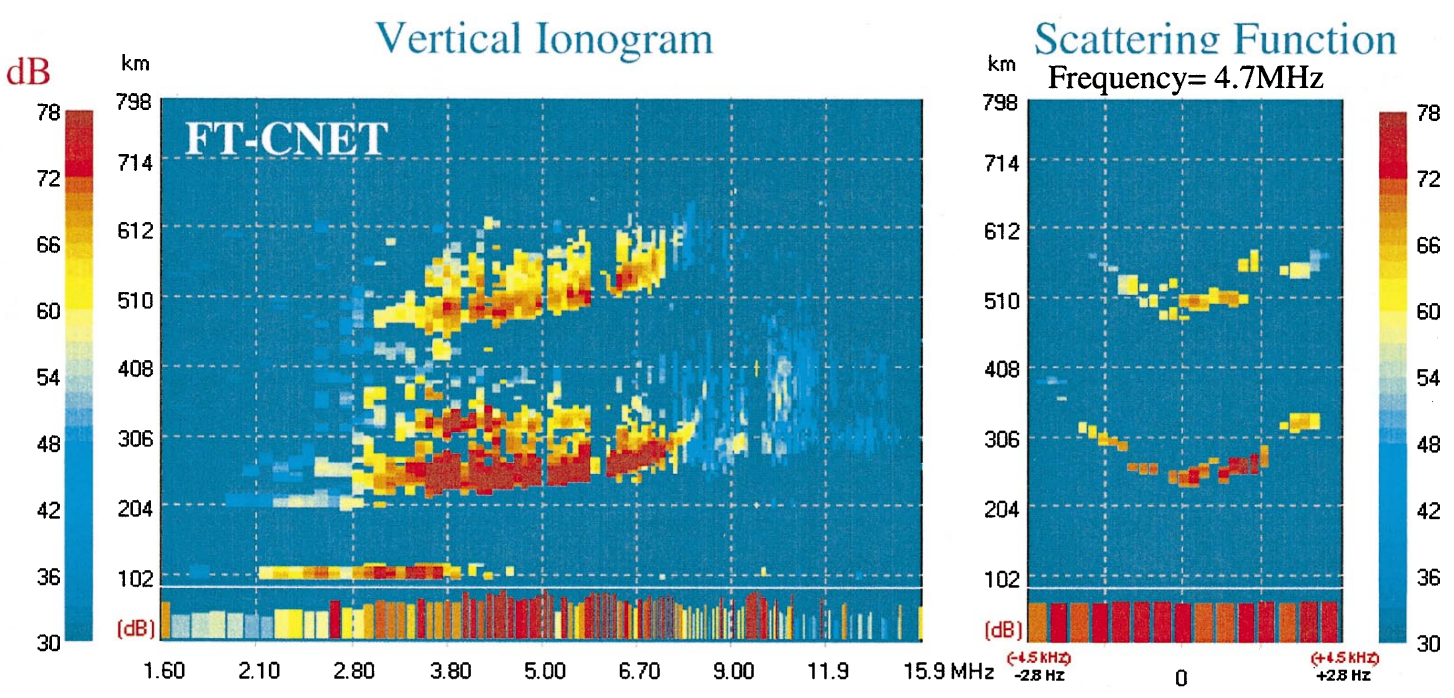

$\mathrm{dB}$
78
72
66
60
54
48
42
36
30
(1)

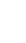

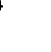



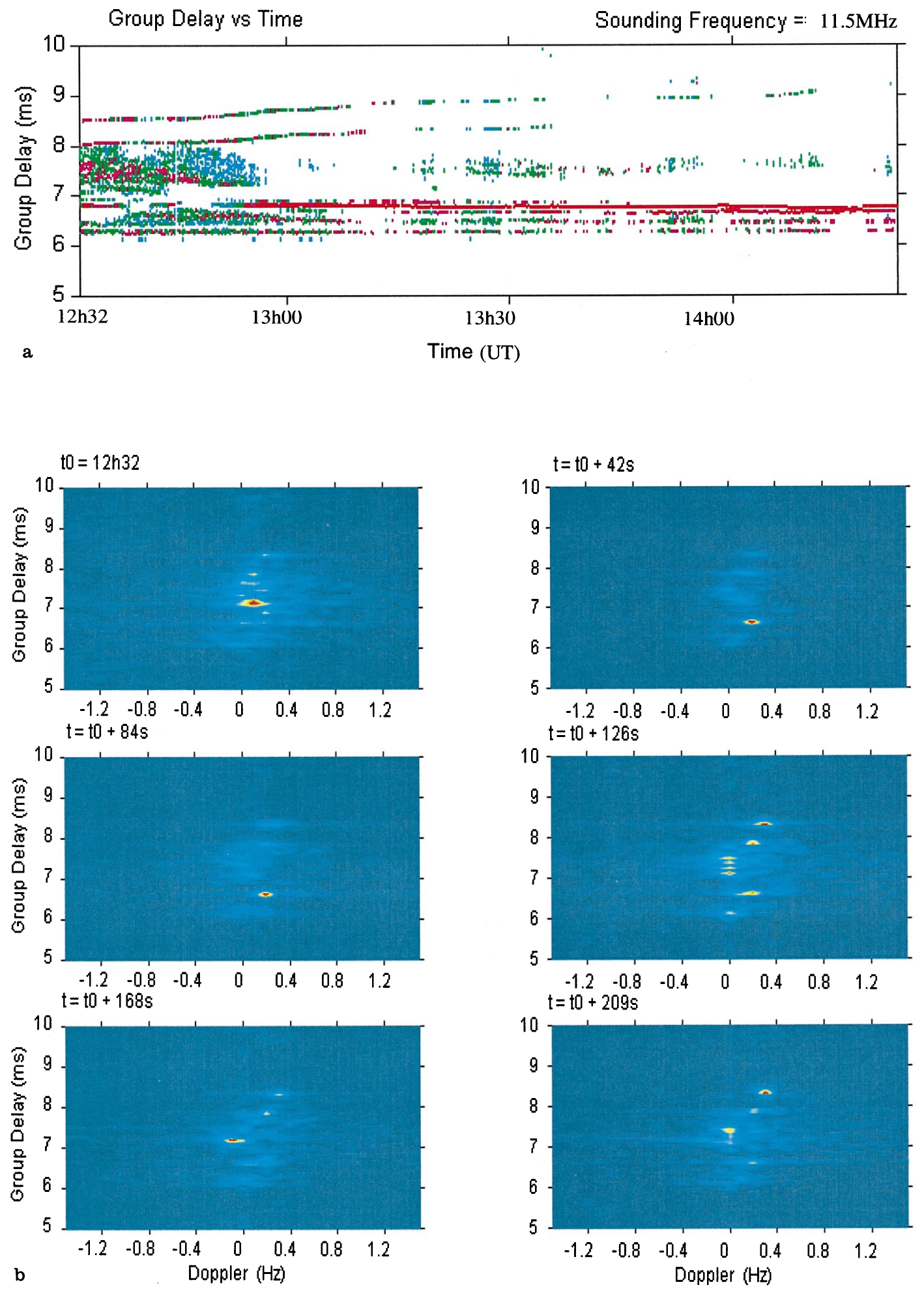

Fig. 3a,b. Experimental analysis of the HF channel working at fixed frequency over a $2000 \mathrm{~km}$ length link between the Ivory-Coast and Senegal on the 21/06/1993. a Group delay evolutions for about

$2 \mathrm{~h}$. b A sequence of scattering functions over a short period from the same experimentation

tions. The results from figure 3 have been obtained at the middle of the day and consequently the Doppler shifts are not very significant. However, Doppler shifts may often be much greater, as at sunrise and sunset for instance.

\section{Conclusion}

We have briefly described in this paper the new CNET SCIPION sounder. It is a new state of the art digital sounder that has been developed for ionospheric monitoring and 
research at both vertical and oblique incidences. A SCIPION system is in the process of being installed in Dakar, Senegal, to study HF propagation in the sub-equatorial ionosphere. However, a prototype version of the sounder has yet been operated there on November 96 and January 97. Some results from these experiments have been shown here. These results indicate clearly that SCIPION permits accurate measurements of the HF channel to be performed while using low transmitted power. It therefore appears that SCIPION is a very well suited tool for studying the $\mathrm{HF}$ channel, in particular at sub-equatorial latitudes where severe propagation conditions are to be found.

Acknowledgements. Topical Editor D. Alcaydé thanks C. Hanceise for his help in evaluating this paper.

\section{References}

Barry, G. H., A low power vertical incidence ionosonde, IEEE Trans. Geosci. Electron, GE-9, 86- 89, 1971.

Bibl, K., and B. W. Reinisch, The universal digital ionosonde, $R a$ dio Sci, 13, 519-530, 1978.
Davies, K., Ionospheric radio propagation, National bureau of standards monographs, Washington, 1965.

Le Roux, Y. M., L. Bertel, J. P. Jolivet, P. Lassudrie-Duchesne, and H. Rouault, Système de mesure des caractéristiques dur canal ionosphérique pour les transmissions numériques, $A G A R D$ CPP, 363, 18.1-18.16, 1984.

Le Roux, Y. M., G. Savidan, G. Du Chaffault, P. Gourvez, and J. P, Jolivet, A combined evaluation and simulation system for the HF channel, IEE ICAP 5th conf., 274, 171-175, 1987.

Le Roux Y. M., M. Niberon, R. Fleury, J. Menard, and J. P. Jolivet, HF channel modeling and simulation, IEE Radio Receivers and Associated Systems 5th conf., 325, 72-76, 1990.

Wright, J. W., and M. L. V. Pittaway, Data processing for the dynasonde: the dopplionogram, J. Geophys. Res., 87, 1589-1598, 1982.

Nickisch, L. J., Non uniform motion and extended media effects on the mutual coherence function, Radio Science, 27-1, 9-22, 1992.

Lauer, C., L. J. Nickish, S. Bottone, and W. Wortman, Equatorial ionospheric irregularity effects for a physic based OTH radar clutter model, I.E.S. 96 Conf., Alexandria, U.S.A., 1B3.1-1B3.7, May 1996.

Wright, J. W., P. E. Argo, and M. L. V. Pittaway, On the radiophysics and geophysics of ionogram spread F, Radio Science, 31-2, 349-366, 1996. 